\title{
Musculoskeletal pathology as an early warning sign of systemic amyloidosis: a systematic review of amyloid deposition and orthopedic surgery
}

Austin E. Wininger', Brian M. Phelps ${ }^{1}$, Jessica T. Le', Joshua D. Harris ${ }^{1}$, Barry H. Trachtenberg ${ }^{2}$ and Shari R. Liberman ${ }^{1 *}$

\begin{abstract}
Background: Transthyretin and immunoglobulin light-chain amyloidoses cause amyloid deposition throughout various organ systems. Recent evidence suggests that soft tissue amyloid deposits may lead to orthopedic conditions before cardiac manifestations occur. Pharmacologic treatments reduce further amyloid deposits in these patients. Thus, early diagnosis improves long term survival.

Questions/purposes: The primary purpose of this systematic review was to characterize the association between amyloid deposition and musculoskeletal pathology in patients with common orthopedic conditions. A secondary purpose was to determine the relationship between amyloid positive biopsy in musculoskeletal tissue and the eventual diagnosis of systemic amyloidosis.

Methods: We performed a systematic review using PRISMA guidelines. Inclusion criteria were level I-IV evidence articles that analyzed light-chain or transthyretin amyloid deposits in common orthopedic surgeries. Study methodological quality, risk of bias, and recommendation strength were assessed using MINORS, ROBINS-I, and SORT.
\end{abstract}

Results: This systematic review included 24 studies for final analysis (3606 subjects). Amyloid deposition was reported in five musculoskeletal pathologies, including carpal tunnel syndrome (transverse carpal ligament and flexor tenosynovium), hip and knee osteoarthritis (synovium and articular cartilage), lumbar spinal stenosis (ligamentum flavum), and rotator cuff tears (tendon). A majority of studies reported a mean age greater than 70 for patients with TTR or AL positive amyloid.

Conclusions: This systematic review has shown the presence of amyloid deposition detected at the time of common orthopedic surgeries, especially in patients $\geq 70$ years old. Subtyping of the amyloid has been shown to enable diagnosis of systemic light-chain or transthyretin amyloidosis prior to cardiac manifestations.

Level of evidence: Level IV.

Keywords: Cardiac amyloidosis, Amyloid, Transthyretin, Immunoglobulin light-chain, Musculoskeletal soft tissue, Orthopedic surgery

\footnotetext{
* Correspondence: drliberman@houstonmethodist.org

${ }^{1}$ Houston Methodist Orthopedics \& Sports Medicine, 6445 Main Street,

Outpatient Center, Suite 2500, Houston, TX 77030, USA

Full list of author information is available at the end of the article
}

C C The Author(s). 2021 Open Access This article is licensed under a Creative Commons Attribution 4.0 International License, which permits use, sharing, adaptation, distribution and reproduction in any medium or format, as long as you give appropriate credit to the original author(s) and the source, provide a link to the Creative Commons licence, and indicate if changes were made. The images or other third party material in this article are included in the article's Creative Commons licence, unless indicated otherwise in a credit line to the material. If material is not included in the article's Creative Commons licence and your intended use is not permitted by statutory regulation or exceeds the permitted use, you will need to obtain permission directly from the copyright holder. To view a copy of this licence, visit http://creativecommons.org/licenses/by/4.0/. The Creative Commons Public Domain Dedication waiver (http://creativecommons.org/publicdomain/zero/1.0/) applies to the data made available in this article, unless otherwise stated in a credit line to the data. 


\section{Introduction}

Amyloidosis is a systemic disease characterized by extracellular deposition of misfolded protein fragments throughout the body. Amyloid proteins can deposit in any tissue or organ, and depending on the location, may lead to dysfunction due to compressive or degenerative pathology $[1,2]$. Two of the most commonly misfolded protein precursors that lead to cardiac manifestations are transthyretin and immunoglobulin light-chain, accounting for $>95 \%$ of cardiac amyloidoses (Table 1). The systemic disease created by these two protein precursors are referred to as transthyretin amyloidosis (ATTR) and immunoglobulin light-chain amyloid amyloidosis (AL). ATTR has two subtypes: a wild type form (ATTRwt) and an inherited, mutant form (ATTRm) [3].

Both ATTR and AL result in varying degrees of extracellular amyloid deposits throughout the body, including the heart, nervous tissue, gastrointestinal tract, and musculoskeletal soft tissues [4, 5]. Cardiac amyloid deposition is the most predictive of morbidity and mortality due to a restrictive cardiomyopathy and subsequent heart failure [6, 7]. Median survival for ATTRwt and ATTRm with cardiac involvement is typically under 60 months [8, 9]. Once amyloid fibrils have deposited within the walls of the heart, it is irreversible. Recent pharmacologic advances for both ATTR and AL have resulted in treatments that can halt amyloid deposition, slow disease progression, decrease morbidity, and increase patient lifespan [9-11].
A significant proportion of patients with AL or ATTR develop amyloid deposition in musculoskeletal soft tissues, including ligaments, tendons, and articular cartilage [12-14]. Several studies have demonstrated that bilateral carpal tunnel syndrome often precedes the diagnosis of cardiac amyloidosis by 5 to 10 years [15-18]. A recent study revealed that $10 \%$ of males over 50 and females over 60 undergoing routine carpal tunnel release had amyloid positive tenosynovial biopsies [19]. Further workup revealed that two were found to have cardiac amyloidosis, one case of AL diagnosed by echocardiography and one case of ATTR by scintigraphy [19]. The prevalence of other musculoskeletal pathologies in the setting of multiorgan amyloidosis has not been well described in the literature. Recent data suggests that in addition to carpal tunnel syndrome, lumbar spinal stenosis may be an early manifestation of cardiac amyloidosis due to amyloid deposition in the ligamentum flavum [20, 21]. Moreover, TTR and light-chain amyloid deposits have been reported in synovial tissue obtained during hip and knee arthroplasties, with one study revealing that patients with ATTR cardiomyopathy were over five times more likely to have undergone total hip arthroplasty than the general population [22-27]. A focused review on the musculoskeletal manifestations of amyloidosis suggested a possible role of histological screening for amyloidosis during common orthopedic surgeries [28]. Current literature is lacking a systematic review on the results of biopsy samples of

Table 1 Most common subtypes of systemic amyloidosis

\begin{tabular}{|c|c|c|c|c|c|}
\hline $\begin{array}{l}\text { Subtype of } \\
\text { Systemic } \\
\text { Amyloidosis }\end{array}$ & ATTRwt & ATTRm & AA & $A L$ & AB2M \\
\hline $\begin{array}{l}\text { Protein } \\
\text { Deposited }\end{array}$ & Transthyretin (wildtype) & Transthyretin (mutated) & Serum Amyloid A & $\begin{array}{l}\text { Immunoglobulin } \\
\text { light chain }\end{array}$ & Beta-2 Microglobulin \\
\hline $\begin{array}{l}\text { Protein } \\
\text { Function and } \\
\text { Source }\end{array}$ & $\begin{array}{l}\text { Thyroxine and retinol binding } \\
\text { protein made in liver and } \\
\text { choroid plexus }\end{array}$ & $\begin{array}{l}\text { Thyroxine and retinol } \\
\text { binding protein made in liver } \\
\text { and choroid plexus }\end{array}$ & $\begin{array}{l}\text { Acute phase protein } \\
\text { made in liver that } \\
\text { accumulates with } \\
\text { sustained and chronic } \\
\text { inflammation }\end{array}$ & $\begin{array}{l}\text { Immunoglobulin } \\
\text { produced by } \\
\text { clonal plasma } \\
\text { cells in the bone } \\
\text { marrow }\end{array}$ & $\begin{array}{l}\text { Component of major } \\
\text { histocompatibility } \\
\text { complex type 1, } \\
\text { which is on all } \\
\text { nucleated cells }\end{array}$ \\
\hline $\begin{array}{l}\text { Other Names } \\
\text { for the Disease }\end{array}$ & $\begin{array}{l}\text { Senile restrictive } \\
\text { cardiomyopathy }\end{array}$ & $\begin{array}{l}\text { Familial cardiomyopathy, } \\
\text { familial neuropathy }\end{array}$ & $\begin{array}{l}\text { Secondary systemic } \\
\text { amyloidosis }\end{array}$ & $\begin{array}{l}\text { Primary systemic } \\
\text { amyloidosis }\end{array}$ & $\begin{array}{l}\text { Dialysis-related } \\
\text { amyloidosis }\end{array}$ \\
\hline $\begin{array}{l}\text { Major Organs } \\
\text { Involved }\end{array}$ & $\begin{array}{l}\text { Heart, musculoskeletal, } \\
\text { nervous }\end{array}$ & $\begin{array}{l}\text { Heart, nervous, } \\
\text { musculoskeletal }\end{array}$ & $\begin{array}{l}\text { Kidney, nervous, heart, } \\
\text { lung }\end{array}$ & $\begin{array}{l}\text { Heart, kidney, } \\
\text { liver, gastro- } \\
\text { intestinal, ner- } \\
\text { vous, lung, soft } \\
\text { tissue }\end{array}$ & $\begin{array}{l}\text { Autonomic nervous, } \\
\text { musculoskeletal }\end{array}$ \\
\hline $\begin{array}{l}\text { Implicated in } \\
\text { Musculoskeletal } \\
\text { Pathology }\end{array}$ & Yes & Yes & Yes & Yes & Yes \\
\hline Treatment & $\begin{array}{l}\text { Novel therapies that decrease } \\
\text { transthyretin production or } \\
\text { stabilize transthyretin to } \\
\text { prevent further amyloid } \\
\text { deposition }\end{array}$ & $\begin{array}{l}\text { Novel therapies that } \\
\text { decrease transthyretin } \\
\text { production or stabilize } \\
\text { transthyretin to prevent } \\
\text { further amyloid deposition }\end{array}$ & $\begin{array}{l}\text { Treatment of } \\
\text { underlying } \\
\text { inflammatory condition }\end{array}$ & $\begin{array}{l}\text { Chemotherapy } \\
\text { directed at } \\
\text { plasma cell clone }\end{array}$ & $\begin{array}{l}\text { High flux } \\
\text { hemodialysis } \\
\text { membrane, renal } \\
\text { transplantation }\end{array}$ \\
\hline
\end{tabular}


musculoskeletal soft tissue in regard to the presence of amyloid and the eventual diagnosis of systemic amyloidosis.

The primary purpose of this systematic review was to characterize the association between amyloid protein deposition and musculoskeletal pathology in patients undergoing common orthopedic surgeries. A secondary purpose was to determine the relationship between amyloid presence detected in musculoskeletal tissue at the time of orthopedic surgery and the eventual diagnosis of systemic amyloidosis. The authors hypothesize that both TTR and immunoglobulin light-chain amyloid would be found in musculoskeletal soft tissue biopsy of patients undergoing orthopedic surgeries and that a small percentage of these patients would eventually be diagnosed with systemic amyloidosis.

\section{Methods}

A systematic review was performed following Preferred Reporting Items for Systematic Reviews and Metaanalyses (PRISMA) guidelines [29]. The authors of this study conducted separate searches of the following databases since their inceptions to present day: PubMed, Scopus, Web of Science, and Google Scholar on March 30, 2020. The main search was performed in PubMed using controlled vocabulary $(\mathrm{MeSH})$ and natural language (title, abstract, and other terms). Search terms focused on (1) amyloid, amyloid deposits, amyloidosis and (2) orthopedics, orthopedic disorders, and musculoskeletal disease (Additional Table 1). These terms were tested for relevancy in PubMed and once finalized, were translated into Scopus, Web of Science, and Google Scholar for article retrieval. All article duplicates were removed prior to screening.

Eligible studies consisted of Level I-IV studies published in the English language prior to March 30, 2020, that investigated light-chain or TTR amyloid deposits in musculoskeletal tissues of patients being treated for common orthopedic conditions. Both print and electronically published journal articles were eligible for inclusion. Screening was performed independently by two reviewers (AEW and BMP) using a prior methodology following PRISMA guidelines (Additional Table 2) [30].

Initial screening of titles and abstracts were performed in Rayyan QCRI using the predetermined inclusion criteria on whether the manuscript discussed the presence of amyloid in tissue samples removed during routine musculoskeletal operations. After initial screening, fulltext articles were assessed for eligibility. Any disagreements at the end of each step were settled by discussion between the two reviewers. In all cases, a consensus was reached. All references within included studies were cross-referenced and assessed for potential inclusion if missed by the initial search. Studies that were not amyloid focused or that focused on secondary causes of amyloidosis, such as beta- 2 microglobulin amyloid related to dialysis or serum amyloid A related to chronic inflammatory conditions, were excluded. Studies that evaluated uncommon orthopedic conditions, such as multiple myeloma, soft-tissue or osseous amyloidoma, and proximal myopathy, were also excluded. Level $\mathrm{V}$ evidence expert opinion, case reports, and letters to editors were excluded. Medical conference abstracts and synthetic review articles (systematic review, meta-analysis, scoping review) were excluded as well. Duplicate subject publications within separate unique studies were not reported more than once. In the situation of duplicate studies from the same author(s) and/or institution(s) reporting on the same or overlapping subjects, only one study was retained (either highest level of evidence, largest number of subjects, longest follow-up, or most pertinent primary outcome score [more explicit reporting of histopathological amyloid findings or with a more thorough examination for concomitant systemic amyloidosis] [or relevant secondary outcome score $\{\mathrm{s}\}$ ]) and the other(s) were excluded.

From each article, details regarding the participants (male/female, mean age, diagnosis) and interventions (surgery, biopsy type, biopsy location, and presence of TTR or light-chain amyloid positive biopsy), outcomes (subjective patient-reported outcomes, objective clinician measured outcomes [motion, strength, mortality/survival]). Study type and design were assessed. Levels I, II, III, and IV of evidence were assigned to studies according to the Oxford Centre for Evidence Based Medicine used by the American version of the Journal of Bone and Joint Surgery [31]. Study-specific demographics, interventions, and biopsy data were extracted from each study.

The methodological quality of the included studies was assessed using the MINORS (methodological index for non-randomized studies) criteria [32]. The risk of bias was evaluated using the ROBINS-I (Risk of Bias in Non-randomized Studies-of Interventions) which assigns an overall bias as low, moderate, severe or critical [33]. Recommendation regarding the quality, quantity, and consistency of evidence was made using SORT (Strength of Recommendation Taxonomy) [34]. These assessments were performed by one author (AEW) and independently checked by another author (BMP).

\section{Results}

Database searches of PubMed, Scopus, Web of Science, and Google Scholar resulted in 3944 unique abstracts. Of the 3944 abstracts screened, 3812 did not meet our inclusion criteria. After screening, 62 full-text articles were assessed for eligibility, with 24 articles included in the final analysis (Fig. 1, Table 2). 


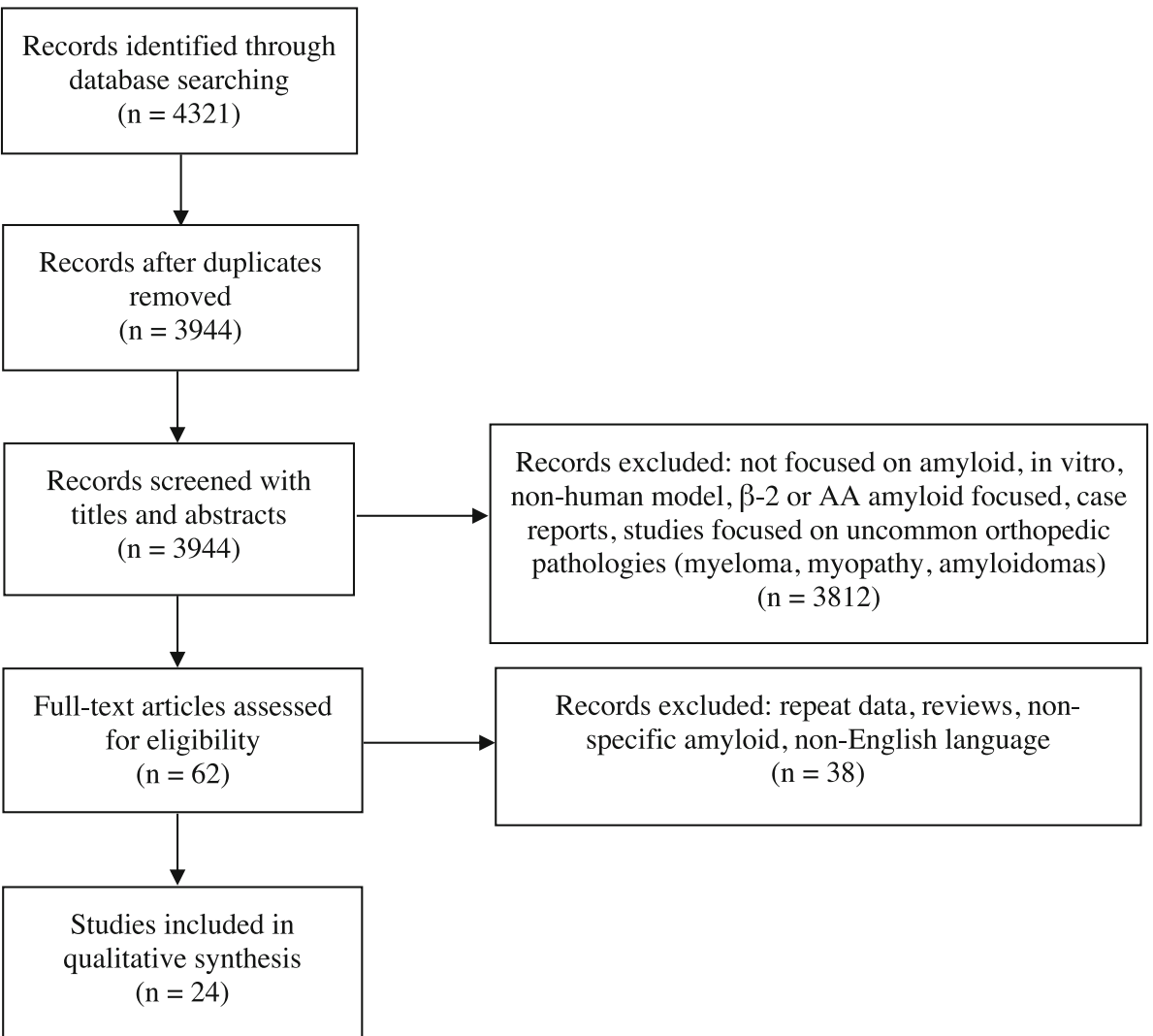

Fig. 1 Flow chart application of exclusion criteria for study inclusion

The 24 studies in this systematic review included a total of 13 cohorts of patients with carpal tunnel syndrome, six with lumbar spinal stenosis, five with knee osteoarthritis, two with hip osteoarthritis, two with rotator cuff pathology, and two with biceps tendon pathology. Nineteen of the included studies reported results from musculoskeletal biopsy samples: 10 from transverse carpal ligament or flexor tenosynovium during carpal tunnel release, four from ligamentum flavum during lumbar decompression, four from synovium and cartilage during total knee arthroplasty, one from synovium and cartilage during total hip arthroplasty, and one from rotator cuff tendon during rotator cuff repair (Table 3).

Immunohistochemical testing was completed to subtype the amyloid with report on the occurrence of TTR or light-chain amyloid deposits (Additional Table 3). Overall, 3606 patients were included across all studies (1625 males, 1956 females). Of these, 2183 patients underwent biopsy of musculoskeletal soft tissue, with 410 TTR positive biopsies and 8 light-chain positive biopsies. Among the 1753 patients $(520$ males, $1071 \mathrm{fe}$ males) who underwent carpal tunnel releases with biopsy. TTR positive biopsies were identified in 241 of these patients and 7 had light-chain positive biopsies. There were 157 patients (81 males, 40 females) who underwent lumbar decompression for spinal stenosis with biopsy. TTR positive biopsies were identified in 64 of these patients and 0 had light-chain positive biopsies. Among the 382 patients (82 males, 294 females) who underwent hip or knee arthroplasty with biopsy, TTR positive biopsies were identified in 97 of these patients and 1 had light-chain positive biopsies (Table 4). A majority of studies reported a mean age greater than 70 for patients with TTR or AL positive amyloid. To diagnose either ATTR or AL after positive biopsy, cardiac workup with electrocardiogram, echocardiography, or scintigraphy and hematology workup with urine and serum monoclonal antibody tests could be considered.

An attempt was made to extract outcomes data from each study, however patient follow-up was poorly reported. Of the patients with amyloid positive amyloid biopsies collected at the time of carpal tunnel release, nine were subsequently diagnosed with systemic amyloidosis (Table 5). Systemic amyloidosis was diagnosed by genetic testing, a second biopsy location, physical examination, electrocardiography, N-terminal pro-B-type natriuretic peptide, troponin $\mathrm{T}$, echocardiography, or 
Table 2 Summary of key elements of assessed studies

\begin{tabular}{|c|c|c|c|c|c|c|c|}
\hline Authors & Year & Journal & Country & $\begin{array}{l}\text { Level of } \\
\text { Evidence }\end{array}$ & $\begin{array}{l}\text { Study } \\
\text { type }\end{array}$ & Study Design & $\begin{array}{l}\text { Length of Follow Up } \\
\text { (months) }\end{array}$ \\
\hline Akasaki et al. [35] & 2015 & Arthritis Rheumatol & $\begin{array}{l}\text { United States } \\
\text { (California) }\end{array}$ & 3 & Diagnostic & Case control & none \\
\hline $\begin{array}{l}\text { aus dem Siepen } \\
\text { et al. [36] }\end{array}$ & 2019 & Clin Res Cardiol & Germany & 3 & Diagnostic & $\begin{array}{l}\text { Retrospective } \\
\text { comparative }\end{array}$ & $29 \pm 29$ \\
\hline Bishop et al. [15] & 2018 & Amyloid & $\begin{array}{l}\text { United States } \\
\text { (Maryland) }\end{array}$ & 3 & Diagnostic & $\begin{array}{l}\text { Retrospective } \\
\text { comparative }\end{array}$ & none \\
\hline $\begin{array}{l}\text { Fernandez Fuertes } \\
\text { et al. [37] }\end{array}$ & 2017 & Med Clin (Barc) & Spain & 3 & Diagnostic & $\begin{array}{l}\text { Prospective } \\
\text { cohort }^{*}\end{array}$ & 36 \\
\hline Geller et al. [38] & 2017 & JAMA & $\begin{array}{l}\text { United States } \\
\text { (Massachusetts) }\end{array}$ & 3 & Diagnostic & Case control & none \\
\hline Gies et al. [39] & 1996 & Clin Neuropathol & Germany & 4 & Diagnostic & Case series & none \\
\hline Gioeva et al. [14] & 2013 & Amyloid & Germany & 4 & Diagnostic & Case series & none \\
\hline Gu et al. [23] & 2014 & J Zhejiang Univ Sci B & China & 3 & Diagnostic & Case control & none \\
\hline Kyle et al. [40] & 1992 & Am J Clin Pathol & $\begin{array}{l}\text { United States } \\
\text { (Minnesota) }\end{array}$ & 4 & Diagnostic & Case series & 168 \\
\hline $\begin{array}{l}\text { Nakamichi et al. } \\
\text { [41] }\end{array}$ & 1996 & Muscle Nerve & Japan & 4 & Diagnostic & Case series & 56 \\
\hline $\begin{array}{l}\text { Niggemeyer et al. } \\
\text { [42] }\end{array}$ & 2011 & Arch Orthop Trauma Surg & Germany & 4 & Diagnostic & Case series & none \\
\hline Rubin et al. [27] & 2017 & Amyloid & $\begin{array}{l}\text { United States (New } \\
\text { York) }\end{array}$ & 3 & Diagnostic & $\begin{array}{l}\text { Retrospective } \\
\text { comparative }\end{array}$ & none \\
\hline Samões et al. [43] & 2017 & Amyloid & Portugal & 4 & Diagnostic & Case series & 38.4 \\
\hline Scott et al. [44] & 2019 & Plast Reconstr Surg & $\begin{array}{l}\text { United States } \\
\text { (Arizona) }\end{array}$ & 4 & Diagnostic & Case series & none \\
\hline Sekijima et al.18 & 2011 & Hum Pathol & Japan & 3 & Diagnostic & Case control & none \\
\hline Sperry et al. [45] & 2018 & J Am Coll Cardiol & United States (Ohio) & 2 & Diagnostic & $\begin{array}{l}\text { Prospective } \\
\text { cohort }\end{array}$ & next phase of study \\
\hline Stein et al. [46] & 1987 & $\begin{array}{l}\text { Virchows Arch A Pathol } \\
\text { Anat Histopathol }\end{array}$ & Germany & 4 & Diagnostic & Case series & none \\
\hline Sueyoshi et al. [13] & 2011 & Hum Pathol & Japan & 4 & Diagnostic & Case series & none \\
\hline $\begin{array}{l}\text { Takanashi et al. } \\
\text { [25] }\end{array}$ & 2013 & Amyloid & Japan & 4 & Diagnostic & Case series & none \\
\hline Uchihara et al. [47] & 2018 & Pathol Res Pract & Japan & 4 & Diagnostic & Case series & none \\
\hline $\begin{array}{l}\text { Westermark et al. } \\
\text { [20] }\end{array}$ & 2014 & Ups J Med Sci & Sweden & 4 & Diagnostic & Case series & none \\
\hline $\begin{array}{l}\text { Yanagisawa et al. } \\
\text { [21] }\end{array}$ & 2015 & Mod Pathol & Japan & 4 & Diagnostic & Case series & none \\
\hline $\begin{array}{l}\text { Yanagisawa et al. } \\
{[26]}\end{array}$ & 2016 & Amyloid & Japan & 4 & Diagnostic & Case series & none \\
\hline $\begin{array}{l}\text { Zegri-Reiriz et al. } \\
\text { [48] }\end{array}$ & 2019 & J Cardiovasc Transl Res & Spain & 3 & Diagnostic & $\begin{array}{l}\text { Prospective } \\
\text { cohort }^{\mathrm{a}}\end{array}$ & none \\
\hline
\end{tabular}

${ }^{a}$ no consistently applied reference standard

technetium pyrophosphate nuclear scintigraphy. No diagnosis of systemic or cardiac amyloidosis was reported in patients with positive biopsies undergoing lumbar decompression, hip, or knee arthroplasty.

Study methodological quality was assessed using MINORS and the risk of bias using ROBINS-I (Table 6). Although these studies indicate that orthopedic pathology can be an early warning sign or early manifestation of cardiac amyloidosis, most only report the histological findings and are of very low methodological quality with critical or serious risk of bias. Twenty of the 24 studies do not include long term follow up or monitor for current or future cardiac manifestations of amyloidosis. As a body of evidence, the included studies are SORT $C$, indicating the recommendation level is based on consensus, usual practice, opinion, or disease-oriented evidence. 
Table 3 Summary of key data and findings of assessed studies

\begin{tabular}{|c|c|c|c|c|c|c|}
\hline Authors & $\begin{array}{l}\text { Total } \\
\text { Subjects }\end{array}$ & $\begin{array}{l}\text { Male/ } \\
\text { Female }\end{array}$ & $\begin{array}{l}\text { Mean } \\
\text { Age } \\
\text { (years) }\end{array}$ & $\begin{array}{l}\text { Orthopedic } \\
\text { Pathology }\end{array}$ & $\begin{array}{l}\text { Biopsy } \\
\text { location }\end{array}$ & Key Findings \\
\hline $\begin{array}{l}\text { Akasaki et al. } \\
\text { [35] }\end{array}$ & 36 & $16 / 20$ & 60.8 & knee OA & cartilage & $\begin{array}{l}\text { Young normal cartilage: } 0 \% \text { amyloid deposits, } 0 \% \text { TTR detected; Aged normal cartilage: } \\
58 \% \text { amyloid deposits, } 83 \% \text { TTR detected; OA cartilage: } 100 \% \text { amyloid deposits, 100\% } \\
\text { TTR detected }\end{array}$ \\
\hline $\begin{array}{l}\text { aus dem } \\
\text { Siepen et al. } \\
{[36]}\end{array}$ & 466 & $373 / 93$ & 63.7 & CTS and LSS & none & $\begin{array}{l}\text { Latency between CTR and diagnosis of systemic amyloidosis was significantly longer in } \\
\text { ATTRwt compared to ATTRm ( } 117 \pm 179 \text { months vs } 66 \pm 73 \text { months; } p=0.02)\end{array}$ \\
\hline $\begin{array}{l}\text { Bishop et al. } \\
{[15]}\end{array}$ & 82 & $55 / 27$ & 70.5 & CTS & none & $\begin{array}{l}\text { CTS associated with a longer delay in diagnosis (OR: 2.13; } 95 \% \text { Cl 1.49-3.03) in } 82 \\
\text { patients with AL or ATTR cardiomyopathy }\end{array}$ \\
\hline $\begin{array}{l}\text { Fernandez } \\
\text { Fuertes et al. } \\
\text { [37] }\end{array}$ & 147 & $31 / 116$ & 58 & CTS & TCL, FTS & $\begin{array}{l}29 \text { of } 147(19.7 \%) \text { of patients undergoing CTR had amyloid positive biopsies; } 4 \text { patients } \\
\text { later developed systemic amyloidosis }\end{array}$ \\
\hline $\begin{array}{l}\text { Geller et al. } \\
\text { [38] }\end{array}$ & 151 & $137 / 14$ & 74.7 & $\begin{array}{l}\text { Distal biceps } \\
\text { rupture }\end{array}$ & none & $\begin{array}{l}\text { 33.3\% [95\% Cl, 24.7-42.9\%] of } 111 \text { patients with ATTRwt cardiomyopathy had ruptured } \\
\text { distal biceps tendon on exam }\end{array}$ \\
\hline Gies et al. [39] & 100 & $60 / 40$ & NS & LSS & $\mathrm{LF}$ & $\begin{array}{l}12 \text { of } 100 \text { specimens from LSS and LDH patients contained amyloid; } 5 \text { contained very } \\
\text { strong TTR presence }\end{array}$ \\
\hline $\begin{array}{l}\text { Gioeva et al. } \\
{[14]}\end{array}$ & 1020 & $\begin{array}{l}351 / \\
669\end{array}$ & 61.8 & CTS & $\mathrm{TCL}$ & $\begin{array}{l}98 \text { biopsies contained TTR amyloid; } \\
70 \text { of } 81 \text { patients with DNA sequencing had wildtype TTR gene }\end{array}$ \\
\hline Gu et al. [23] & 36 & $16 / 20$ & 66.4 & knee OA & $\begin{array}{l}\text { knee } \\
\text { synovium }\end{array}$ & $\begin{array}{l}9 \text { of } 36 \text { knee OA patients had amyloid positive biopsies; } \\
8 \text { contained TTR amyloid and } 1 \text { contained light-chain amyloid }\end{array}$ \\
\hline Kyle et al. [40] & 35 & $23 / 12$ & 71 & CTS & TCL, FTS & 33 of 35 had TTR amyloid; 2 developed systemic amyloidosis \\
\hline $\begin{array}{l}\text { Nakamichi } \\
\text { et al. [41] }\end{array}$ & 108 & $5 / 103$ & 56 & CTS & $\mathrm{TCL}, \mathrm{FTS}$ & 10 of 108 patients had amyloid deposits, 6 contained TTR amyloid \\
\hline $\begin{array}{l}\text { Niggemeyer } \\
\text { et al. [42] }\end{array}$ & 50 & $14 / 36$ & 68.4 & Hip OA & $\begin{array}{l}\text { synovium, } \\
\text { cartilage }\end{array}$ & 17 of 50 consecutive patients had amyloid deposits, all contained TTR amyloid \\
\hline $\begin{array}{l}\text { Rubin et al. } \\
{[27]}\end{array}$ & 313 & $234 / 79$ & 66.6 & $\begin{array}{l}\text { knee and hip } \\
\text { OA, rotator cuff }\end{array}$ & none & $\begin{array}{l}\text { THA and TKA significantly more common among ATTR patients with cardiomyopathy } \\
\text { (THA: RR 5.61, 95\% Cl 2.25-4.64; TKA: RR 3.32, 95\% Cl 2.25-4.64) }\end{array}$ \\
\hline $\begin{array}{l}\text { Samões et al. } \\
{[43]}\end{array}$ & 16 & $3 / 13$ & 46.1 & CTS & $\mathrm{TCL}$ & $\begin{array}{l}\text { In } 16 \text { patients with known ATTRm, } 15 \text { patients had CTS that preceded amyloidosis and } \\
14 \text { had amyloid positive biopsies }\end{array}$ \\
\hline $\begin{array}{l}\text { Scott et al. } \\
{[44]}\end{array}$ & 35 & $16 / 19$ & 72 & recurrent CTS & FTS & $\begin{array}{l}9 \text { of } 35 \text { patients (26\%) with recurrent CTS had an amyloid positive biopsy, } 7 \text { of which } \\
\text { contained TTR }\end{array}$ \\
\hline $\begin{array}{l}\text { Sekijima et al. } \\
\text { [18] }\end{array}$ & 132 & $40 / 92$ & 71.8 & CTS & FTS & $\begin{array}{l}34 \text { of } 100 \text { patients with idiopathic CTS had TTR amyloid deposits versus } 7 \text { of } 32 \text { autopsy } \\
\text { controls (OR, 15.8; 95\% Cl, 3.3-75.7) }\end{array}$ \\
\hline $\begin{array}{l}\text { Sperry et al. } \\
{[45]}\end{array}$ & 98 & $51 / 47$ & 68.5 & CTS & FTS & $\begin{array}{l}10 \text { of } 98 \text { patients undergoing CTR had an amyloid positive biopsy (7 TTR, } 2 \text { light chain); } \\
3 \text { diagnosed with systemic amyloidosis }\end{array}$ \\
\hline Stein et al. [46] & 108 & NS & NS & CTS & $\mathrm{TCL}$ & $\begin{array}{l}\text { Amyloid deposits were found in } 23 \text { of } 108 \text { (21\%) patients with idiopathic CTS; TTR was } \\
\text { identified in } 14 \text { of these } 23 \text { patients }\end{array}$ \\
\hline $\begin{array}{l}\text { Sueyoshi et al. } \\
\text { [13] }\end{array}$ & 111 & $56 / 55$ & 62 & $\begin{array}{l}\text { CTS, LSS, } \\
\text { rotator cuff }\end{array}$ & FTS, LF, RCT & 39 of 111 specimens contained TTR amyloid deposits \\
\hline $\begin{array}{l}\text { Takanashi } \\
\text { et al. [25] }\end{array}$ & 232 & $31 / 201$ & 73 & knee OA & synovium & 21 of 232 knee OA patients (9\%) had TTR amyloid deposits \\
\hline $\begin{array}{l}\text { Uchihara et al. } \\
\text { [47] }\end{array}$ & 25 & NS & NS & NS & $\begin{array}{l}\text { periarticular } \\
\text { F\&A }\end{array}$ & 3 of 25 specimens contained TTR amyloid \\
\hline $\begin{array}{l}\text { Westermark } \\
\text { et al. [20] }\end{array}$ & 26 & $13 / 13$ & 66.5 & LSS & $\begin{array}{l}\mathrm{LF} \text {, bone } \\
\text { fragments }\end{array}$ & $\begin{array}{l}21 \text { of } 26 \text { specimens contained amyloid deposits; } 5 \text { of } 15 \text { specimens suitable for } \\
\text { immunohistochemistry contained TTR amyloid }\end{array}$ \\
\hline $\begin{array}{l}\text { Yanagisawa } \\
\text { et al. [21] }\end{array}$ & 95 & $68 / 27$ & 70.7 & LSS & LF & $\begin{array}{l}\text { All } 95 \text { LF specimens resected from LSS patients contained amyloid deposits; } 43 \\
\text { contained TTR amyloid }\end{array}$ \\
\hline $\begin{array}{l}\text { Yanagisawa } \\
\text { et al. [26] }\end{array}$ & 52 & $17 / 35$ & 66.6 & knee OA & $\begin{array}{l}\text { periarticular } \\
\text { knee }\end{array}$ & $\begin{array}{l}\text { TTR amyloid deposits were found in specimens from: } 18 \text { of } 51 \text { menisci, } 8 \text { of } 27 \text { articular } \\
\text { cartilage, and } 6 \text { of } 34 \text { synovium }\end{array}$ \\
\hline $\begin{array}{l}\text { Zegri-Reiriz } \\
\text { et al. [48] }\end{array}$ & 101 & $32 / 69$ & 69 & CTS & $\begin{array}{l}\text { none, } \\
\text { myocardial }\end{array}$ & $\begin{array}{l}\text { Prevalence of cardiac amyloidosis in the cohort was } 1.2 \%(3 / 233) \text { and } 5.5 \%(3 / 55) \text { for } \\
\text { patients with LVH and bilateral CTS }\end{array}$ \\
\hline
\end{tabular}

Cl confidence interval, CTS carpal tunnel syndrome, DNA deoxyribonucleic acid, F\&A foot and ankle, FTS flexor tenosynovium, $L F$ ligamentum flavum, $L D H$ lumbar disc herniation, $L S S$ lumbar spinal stenosis, $L V H$ left ventricular hypertrophy, NS not specified, $O A$ osteoarthritis, $O R$ odds ratio, $R C T$ rotator cuff tendon, $R R$ relative risk, TCL transverse carpal ligament, THA total hip arthroplasty, TKA total knee arthroplasty

\section{Discussion}

This systematic review supports the author's hypotheses that amyloid deposits are present within musculoskeletal soft tissues encountered during common orthopedic surgeries and identifying their presence can aid in diagnosing ATTR or AL. This systematic review also supports that histological testing of orthopedic biopsy samples may enable screening for unsuspected systemic 
Table 4 The incidence of TTR and immunoglobulin light-chain amyloid from tissue samples removed during common orthopedic operations

\begin{tabular}{lllllll}
\hline & $\begin{array}{l}\text { Total } \\
\text { Patients }\end{array}$ & $\begin{array}{l}\text { Male/Female (when } \\
\text { specified) }\end{array}$ & $\begin{array}{l}\text { Patients with } \\
\text { Orthopedic Biopsy }\end{array}$ & $\begin{array}{l}\text { TTR + } \\
\text { Biopsy }\end{array}$ & $\begin{array}{l}\text { Ig Light-Chain } \\
\text { + Biopsy }\end{array}$ & $\begin{array}{l}\text { \% of patients with TTR or } \\
\text { light-chain+ Biopsy }\end{array}$ \\
\hline All Studies & 3606 & $1625 / 1956$ & 2183 & 410 & 8 & $19.1 \%$ \\
CTS with Biopsy & 1753 & $520 / 1071$ & 1753 & 241 & 7 & $14.1 \%$ \\
$\begin{array}{l}\text { LSS with Biopsy } \\
\text { a }\end{array}$ & 157 & $81 / 40$ & 157 & 64 & 0 & $40.8 \%$ \\
$\begin{array}{l}\text { Hip and Knee OA } \\
\text { with Biopsy }\end{array}$ & 382 & $82 / 294$ & 382 & 97 & 1 & $25.7 \%$
\end{tabular}

CTS carpal tunnel syndrome, Ig immunoglobulin, LSS lumbar spinal stenosis, \% percentage, + positive, OA osteoarthritis

${ }^{a}$ Excludes Gies et al. study due to the authors not differentiating lumbar spinal stenosis patients from lumbar disc herniation patients

amyloidosis. Despite the heterogeneity of included studies, the reported findings suggest that ATTR and AL can lead to amyloid deposits that are present in locations of musculoskeletal pathology. TTR amyloid was much more commonly observed than light-chain amyloid within orthopedic biopsies (Table 4).

For carpal tunnel syndrome, the data from Sperry et al. lead Donnelly et al. to propose an algorithm for treating patients undergoing carpal tunnel release [19, 45]. In this, men over the age of 50 and women over the age of 60 with bilateral symptoms or prior release are designated to be in tier 1 . History of spinal stenosis, biceps tendon rupture, atrial fibrillation/flutter, pacemaker, heart failure, or family history of amyloidosis are tier 2. If a patient meets the criteria for tier 1 and has one or more risk factors listed in tier 2, the algorithm recommends a biopsy, amyloid subtyping, and referral to amyloid specialist if found to be positive for TTR or light-chain. Patients presenting with recurrent carpal tunnel syndrome in the setting of true idiopathic disease with no obvious risk factors may be at higher risk for having amyloid deposits within their transverse carpal ligament and flexor tenosynovium [44]. This amyloid was subtyped as TTR deposits in several patient cohorts, but no true prospective follow-up for the development of cardiac amyloidosis has been performed.

Several studies report an association between lumbar spinal stenosis and TTR amyloid deposits within the ligamentum flavum. Both thickened ligamentum flavum and increased lumbar spine instability were associated with a greater quantity of TTR amyloid deposits in spinal stenosis patients [21]. Elderly men with spinal stenosis have been labeled as the most at risk for ATTR and with current evidence may warrant a biopsy [20, 49]. In patients undergoing surgery for lumbar disc herniation, with no additional risk factors for amyloidosis, the literature indicates that amyloid deposition is most often absent from biopsy tissues [21,39].

Studies on hip and knee arthroplasty related to cardiac amyloidosis have indicated the presence of TTR and light-chain amyloid in hip and knee synovium. Whether the amyloid deposition in the synovium is age associated and found by coincidence, or corresponds to a diagnosis of systemic disease, has not been elucidated [27, 42].

Regarding other orthopedic pathology, one study revealed a high occurrence of distal biceps tendon rupture in patients diagnosed with ATTRwt cardiomyopathy [38]. A study reporting the results of rotator cuff biopsies did not provide information beyond the presence of TTR amyloid [13]. In a similar manner, the only foot and ankle study reporting on the presence of TTR amyloid, did not provide any clinical correlation with the positive biopsies [47]. Without a history of carpal tunnel syndrome or lumbar spinal stenosis, biopsy may not be warranted with these pathologies unless there is a red flag in the patient's medical history.

Systemic amyloidosis may be more common than previously recognized $[50,51]$ and current pharmacologic options do not reverse amyloid deposition [52]. For ATTR, three treatments recently received FDA approval, and for $\mathrm{AL}$, improvements in chemotherapy have markedly improved survival, even in cases with cardiac involvement $[1,53,54]$. ATTR and AL are difficult diseases to diagnose due to nonspecific symptoms, overlapping diagnoses, and under-recognition by physicians. As patients could potentially present with musculoskeletal conditions prior to systemic manifestations, orthopedic surgeons may play a role in early diagnosis.

\section{Limitations}

Limitations of this systematic review include predominantly retrospective level-III/IV evidence included for review. The heterogeneous and limited data on gender, age, clinical outcomes, or histopathological findings were unable to be quantitatively assimilated, precluding metaanalysis. Current studies contain little or no follow-up information or monitoring of patients for development of systemic disease or restrictive cardiomyopathy. Moreover, few studies utilized genetic testing and no studies evaluated for serum testing that could rule in or out the need for biopsy pre-operatively. There is a lack of data regarding various musculoskeletal soft tissues of common orthopedic pathology, such as Achilles tendon, 
Table 5 Results of studies reporting on biopsies taken during carpal tunnel release of seemingly idiopathic carpal tunnel syndrome

\begin{tabular}{|c|c|c|c|c|c|}
\hline Study & $\begin{array}{l}\text { Patients with } \\
\text { Biopsy }\end{array}$ & $\begin{array}{l}\text { Amyloid } \\
\text { Positive Biopsy }\end{array}$ & Follow Up & $\begin{array}{l}\text { Diagnosed with } \\
\text { Systemic Amyloidosis }\end{array}$ & $\begin{array}{l}\text { Type of Systemic } \\
\text { Amyloidosis }\end{array}$ \\
\hline Fernandez Fuertes et al. [37] & 147 & 29 & 36 months & 4 & $3 \mathrm{AL}, 1 \mathrm{ATTR}$ \\
\hline Kyle et al. [40] & 35 & 35 & 11 years & 2 & 1 ATTR, 1 untyped \\
\hline Gioeva et al. [14] & 1010 & 98 & None & NR & \\
\hline Nakamichi et al. [41] & 108 & 10 & 12.5 years & 0 & \\
\hline Sekijima et al. [18] & 100 & 34 & None & NR & \\
\hline Sperry et al. [45] & 98 & 10 & $\begin{array}{l}\text { None, concomitant workup for } \\
\text { systemic disease if biopsy positive }\end{array}$ & 3 & 2 ATTR, 1 AL \\
\hline Stein et al. [46] & 108 & 19 & None & NR & \\
\hline Sueyoshi et al. [13] & 54 & 20 & None & NR & \\
\hline
\end{tabular}

patellar tendon, and hip labrum. There is also a lack of data regarding osteoarthritic joints other than the hip and knee. The limitations of any systematic review are a result of the studies they include and analyze. As such, the above-mentioned shortcomings may limit the fidelity of clinical relevance.

\section{Future research directions}

There have been no prospective studies that evaluate all patients regardless of biopsy results for underlying amyloidosis. The results of such a study would enable a more robust analysis of the diagnostic potential of orthopedic biopsies for cardiac amyloidosis. No studies have

Table 6 Critical appraisal of included studies using MINORS and ROBINS-I

\begin{tabular}{|c|c|c|c|}
\hline Study & $\begin{array}{l}\text { MINORS (non-comparative } \\
\text { study, out of } 16 \text { ) }\end{array}$ & $\begin{array}{l}\text { MINORS (comparative } \\
\text { study, out of 24) }\end{array}$ & $\begin{array}{l}\text { ROBINS-I (across } \\
\text { all domains) }\end{array}$ \\
\hline Akasaki et al. [35] & & 12 & serious \\
\hline aus dem Siepen et al. [36] & 7 & & critical \\
\hline Bishop et al. [15] & 6 & & critical \\
\hline Fernandez Fuertes et al. [37] & 11 & & critical \\
\hline Geller et al. [38] & & 12 & critical \\
\hline Gies et al. [39] & 8 & & critical \\
\hline Gioeva et al. [14] & & 6 & critical \\
\hline Gu et al. [23] & & 12 & serious \\
\hline Kyle et al. [40] & 8 & & moderate \\
\hline Nakamichi et al. [41] & 10 & & serious \\
\hline Niggemeyer et al. [42] & 8 & & critical \\
\hline Rubin et al. [27] & & 10 & serious \\
\hline Samões et al. [43] & 9 & & moderate \\
\hline Scott et al. [44] & 4 & & critical \\
\hline Sekijima et al. [18] & & 15 & serious \\
\hline Sperry et al. [45] & 10 & & moderate \\
\hline Stein et al. [46] & 6 & & critical \\
\hline Sueyoshi et al. [13] & 5 & & critical \\
\hline Takanashi et al. [25] & 8 & & moderate \\
\hline Uchihara et al. [47] & 2 & & critical \\
\hline Westermark et al. [20] & 6 & & serious \\
\hline Yanagisawa et al. [21] & 4 & & serious \\
\hline Yanagisawa et al. [26] & 6 & & serious \\
\hline Zegri-Reiriz et al. [48] & 8 & & moderate \\
\hline
\end{tabular}


evaluated the economic implications of a musculoskeletal soft tissue biopsy at the time of orthopedic procedure, but prior authors have indicated that the low cost of screening may avoid the expense of treating progressive heart failure [19]. Future studies should look at the economic impact of a larger testing protocol.

\section{Conclusion}

This systematic review supports the presence of amyloid deposition detected at the time of common orthopedic surgeries, most commonly in patients $\geq 70$ years old. Subtyping of the amyloid can enable diagnosis of lightchain or TTR amyloidosis prior to cardiac manifestations. Patient characteristics that may lead orthopedic surgeons to consider the potential need for amyloid biopsy include: a family member with amyloidosis, a personal history of unexplained peripheral neuropathy or autonomic dysfunction, atrial fibrillation, heart failure, or pacemaker with no definitive cause, or an orthopedic history involving bilateral carpal tunnel syndrome, lumbar spinal stenosis, or multiple atraumatic tendon ruptures. Further prospective studies are needed to better determine when and in what musculoskeletal tissues there may be clinical benefit of biopsy for amyloidosis.

\section{Supplementary Information}

The online version contains supplementary material available at https://doi. org/10.1186/s12891-020-03912-z.

Additional file 1.

Additional file 2.

Additional file 3.

\begin{abstract}
Abbreviations
AL: Immunoglobulin light-chain amyloid amyloidosis; ATTR: Transthyretin amyloidosis; ATTRm: Mutant transthyretin amyloidosis; ATTRwt: Wild type transthyretin amyloidosis; MeSH: Medical subject headings;

MINORS: Methodological index for non-randomized studies; PRIS MA: Preferred reporting items for systematic reviews and meta-analyses; ROBINS-I: Risk of bias in non-randomized studies-of interventions; SORT: Strength of recommendation taxonomy; TTR: Transthyretin
\end{abstract}

\section{Acknowledgements}

None.

\begin{abstract}
Authors' contributions
AEW, JDH, BHT, and SRL contributed to the research design and data interpretation. AEW, BMP, and JTL contributed to the literature search, data collection, and data analysis. AEW, BMP, and JTL drafted the manuscript. AEW, JDH, BHT, and SRL critically revised the manuscript. All authors read and approved the final manuscript.
\end{abstract}

\section{Funding}

The authors received no financial support for the research, authorship, and/ or publication of this article.

\section{Availability of data and materials}

All data generated or analyzed during this study are included in this published article.
Ethics approval and consent to participate

Not applicable.

\section{Consent for publication}

All authors have agreed to the publication.

\section{Competing interests}

AEW, BMP, JTL, and BHT declare they have no conflicts of interests. SRL does not have disclosures relevant to the current study, but does report Acumed: educational support.

JDH does not have disclosures relevant to the current study, but does report AAOS: Board or committee member; American Orthopaedic Society for

Sports Medicine: Board or committee member; Arthrex: Educational support; Arthroscopy: Editorial or governing board; Arthroscopy Association of North America: Board or committee member; DePuy, A Johnson \& Johnson Company: Research support; International Society of Arthroscopy, Knee Surgery, and Orthopaedic Sports Medicine: Board or committee member; Medinc of Texas: Educational support; SLACK Incorporated: Publishing royalties, financial or material support; Smith \& Nephew: Paid consultant, Paid presenter or speaker, Research support; Xodus Medical: Paid presenter or speaker.

\section{Author details}

${ }^{1}$ Houston Methodist Orthopedics \& Sports Medicine, 6445 Main Street, Outpatient Center, Suite 2500, Houston, TX 77030, USA. ${ }^{2}$ Houston Methodist DeBakey Heart \& Vascular Center, 6550 Fannin Street, Smith Tower, Suite 1901, Houston, TX 77030, USA.

Received: 12 October 2020 Accepted: 23 December 2020

Published online: 08 January 2021

\section{References}

1. Siddiqi OK, Ruberg FL. Cardiac amyloidosis: an update on pathophysiology, diagnosis, and treatment. Trends Cardiovasc Med. 2018;28(1):10-21.

2. $\quad$ Sipe JD, Benson MD, Buxbaum JN, et al. Nomenclature 2014: amyloid fibril proteins and clinical classification of the amyloidosis. Amyloid. 2014;21(4): 221-4.

3. Sperry BW, Vranian MN, Hachamovitch R, et al. Subtype-specific interactions and prognosis in cardiac amyloidosis. J Am Heart Assoc. 2016;5(3):e002877.

4. Maurer MS, Elliott P, Comenzo R, et al. Addressing common questions encountered in the diagnosis and Management of Cardiac Amyloidosis. Circulation. 2017;135(14):1357-77.

5. Wechalekar AD, Gillmore JD, Hawkins PN. Systemic amyloidosis. Lancet. 2016;387(10038):2641-54.

6. Maurer MS, Hanna M, Grogan M, et al. Genotype and phenotype of Transthyretin cardiac amyloidosis: THAOS (Transthyretin amyloid outcome survey). J Am Coll Cardiol. 2016;68(2):161-72.

7. Rapezzi C, Merlini G, Quarta CC, et al. Systemic cardiac amyloidoses: disease profiles and clinical courses of the 3 main types. Circulation. 2009;120(13): 1203-12.

8. Lane T, Fontana M, Martinez-Naharro A, et al. Natural history, quality of life, and outcome in cardiac Transthyretin amyloidosis. Circulation. 2019;140(1): $16-26$.

9. Grogan M, Dispenzieri A, Gertz MA. Light-chain cardiac amyloidosis: strategies to promote early diagnosis and cardiac response. Heart. 2017; 103(14):1065-72.

10. Sperry BW, Tang WHW. Amyloid heart disease: genetics translated into disease-modifying therapy. Heart. 2017;103(11):812-7.

11. Vranian MN, Sperry BW, Valent J, Hanna M. Emerging advances in the Management of Cardiac Amyloidosis. Curr Cardiol Rep. 2015;17(11):100.

12. Prokaeva T, Spencer B, Kaut M, et al. Soft tissue, joint, and bone manifestations of $\mathrm{AL}$ amyloidosis: clinical presentation, molecular features, and survival. Arthritis Rheum. 2007;56(11):3858-68.

13. Sueyoshi $\mathrm{T}$, Ueda $\mathrm{M}$, Jono $\mathrm{H}$, et al. Wild-type transthyretin-derived amyloidosis in various ligaments and tendons. Hum Pathol. 2011;42(9): 1259-64.

14. Gioeva Z, Urban P, Meliss RR, et al. ATTR amyloid in the carpal tunnel ligament is frequently of wildtype transthyretin origin. Amyloid. 2013;20(1): $1-6$.

15. Bishop E, Brown EE, Fajardo J, et al. Seven factors predict a delayed diagnosis of cardiac amyloidosis. Amyloid. 2018;25(3):174-9. 
16. Kyle RA, Eilers SG, Linscheid RL, Gaffey TA. Amyloid localized to tenosynovium at carpal tunnel release. Natural history of 124 cases. Am J Clin Pathol. 1989;91(4):393-7.

17. Nakagawa M, Sekijima Y, Yazaki M, et al. Carpal tunnel syndrome: a common initial symptom of systemic wild-type ATTR (ATTRwt) amyloidosis. Amyloid. 2016:23(1):58-63.

18. Sekijima Y, Uchiyama S, Tojo K, et al. High prevalence of wild-type transthyretin deposition in patients with idiopathic carpal tunnel syndrome: a common cause of carpal tunnel syndrome in the elderly. Hum Pathol. 2011:42(11):1785-91.

19. Donnelly JP, Hanna M, Sperry BW, Seitz WH. Carpal tunnel syndrome: a potential early, red-flag sign of amyloidosis. J Hand Surg Am. 2019;44(10): 868-76.

20. Westermark P, Westermark GT, Suhr OB, Berg S. Transthyretin-derived amyloidosis: probably a common cause of lumbar spinal stenosis. Ups J Med Sci. 2014;119(3):223-8.

21. Yanagisawa A, Ueda M, Sueyoshi T, et al. Amyloid deposits derived from transthyretin in the ligamentum flavum as related to lumbar spinal canal stenosis. Mod Pathol. 2015;28(2):201-7.

22. Goffin YA. The association of amyloid deposits and osteoarthritis. Arthritis Rheum. 1983:26(1):120

23. Gu YJ, Ge P, Mu Y, Lu JH, Zheng F, Sun XG. Clinical and laboratory characteristics of patients having amyloidogenic transthyretin deposition in osteoarthritic knee joints. J Zhejiang Univ Sci B. 2014;15(1):92-9.

24. Ladefoged C. Amyloid deposits in the knee joint at autopsy. Ann Rheum Dis. 1986;45(8):668-72.

25. Takanashi T, Matsuda M, Yazaki M, et al. Synovial deposition of wild-type transthyretin-derived amyloid in knee joint osteoarthritis patients. Amyloid. 2013;20(3):151-5.

26. Yanagisawa A, Ueda M, Sueyoshi T, et al. Knee osteoarthritis associated with different kinds of amyloid deposits and the impact of aging on type of amyloid. Amyloid. 2016;23(1):26-32.

27. Rubin J, Alvarez J, Teruya S, et al. Hip and knee arthroplasty are common among patients with transthyretin cardiac amyloidosis, occurring years before cardiac amyloid diagnosis: can we identify affected patients earlier? Amyloid. 2017;24(4):226-30.

28. Nguyen TX, Naqvi A, Thompson TL, Wilson RH. Musculoskeletal manifestations of amyloidosis: a focused review. J Surg Orthop Adv. 2018:27(1):1-5.

29. Moher D, Liberati A, Tetzlaff J, et al. Preferred reporting items for systematic reviews and meta-analyses: the PRISMA statement. Br Med J. 2009;339:b2535.

30. Harris JD, Quatman CE, Manring MM, et al. How to write a systematic review. Am J Sports Med. 2014;42(11):2761-8.

31. Marx RG, Wilson SM, Swiontkowski MF. Updating the assignment of levels of evidence. J Bone Joint Surg Am. 2015;97(1):1-2.

32. Slim K, Nini E, Forestier D, et al. Methodological index for non-randomized studies (minors): development and validation of a new instrument. ANZ J Surg. 2003;73(9):712-6

33. Sterne JA, Hernán MA, Reeves BC, et al. ROBINS-I: a tool for assessing risk of bias in non-randomised studies of interventions. BMJ. 2016:355:14919.

34. Ebell MH, Siwek J, Weiss BD, et al. Strength of recommendation taxonomy (SORT): a patient-centered approach to grading evidence in the medical literature. J Am Board Fam Pract. 2004;17(1):59-67.

35. Akasaki Y, Reixach N, Matsuzaki T, et al. Transthyretin deposition in articular cartilage: a novel mechanism in the pathogenesis of osteoarthritis. Arthritis Rheumatol. 2015;67(8):2097-107.

36. Aus dem siepen F, Hein S, Prestel S, et al. Carpal tunnel syndrome and spinal canal stenosis: harbingers of transthyretin amyloid cardiomyopathy? Clin Res Cardiol. 2019;108(12):1324-30.

37. Fuertes JF, Vicente ÓR, Herráez SS, Pascua LR. Early diagnosis of systemic amyloidosis by means of a transverse carpal ligament biopsy carried out during carpal tunnel syndrome surgery. Med Clin (Barc). 2017;148(5):211-4

38. Geller HI, Singh A, Alexander KM, et al. Association between ruptured distal biceps tendon and wild-type Transthyretin cardiac amyloidosis. JAMA. 2017; 318(10):962-3.

39. Gies U, Linke RP, Schachenmayr W. Amyloid deposits of immunohistochemically different classes in the ligamentum flavum in biopsies from patients with herniated discs or lumbar spinal stenosis. Clin Neuropathol. 1996;15(1):54-9.

40. Kyle RA, Gertz MA, Linke RP. Amyloid localized to tenosynovium at carpal tunnel release. Immunohistochemical identification of amyloid type. Am J Clin Pathol. 1992;97(2):250-3.
41. Nakamichi Kl, Tachibana S. Amyloid deposition in the synovium and ligament in idiopathic carpal tunnel syndrome. Muscle Nerve. 1996;19(10): 1349-51.

42. Niggemeyer $\mathrm{O}$, Steinhagen J, Deuretzbacher $\mathrm{G}$, et al. Amyloid deposition in osteoarthritis of the hip. Arch Orthop Trauma Surg. 2011;131(5):637-43.

43. Samões R, Taipa R, Valdrez K, et al. Amyloid detection in the transverse carpal ligament of patients with hereditary ATTR V30M amyloidosis and carpal tunnel syndrome. Amyloid. 2017;24(2):73-7.

44. Scott KL, Conley CR, Renfree KJ. Histopathologic evaluation of flexor Tenosynovium in recurrent carpal tunnel syndrome. Plast Reconstr Surg. 2019;143(1):169-75

45. Sperry BW, Reyes BA, Ikram A, et al. Tenosynovial and cardiac amyloidosis in patients undergoing carpal tunnel release. J Am Coll Cardiol. 2018;72(17): 2040-50.

46. Stein K, Störkel S, Linke RP, Goebel HH. Chemical heterogeneity of amyloid in the carpal tunnel syndrome. Virchows Arch A Pathol Anat Histopathol. 1987:412(1):37-45.

47. Uchihara Y, Iwata E, Papadimitriou-olivgeri I, et al. Localised foot and ankle amyloid deposition. Pathol Res Pract. 2018;214(10):1661-6.

48. Zegri-reiriz I, de Daro-Del Moral FJ, Dominguez F, et al. Prevalence of cardiac amyloidosis in patients with carpal tunnel syndrome. J Cardiovasc Transl Res. 2019:12(6):507-13.

49. Brunjes DL, Castano A, Clemons A, et al. Transthyretin cardiac amyloidosis in older Americans. J Card Fail. 2016;22(12):996-1003.

50. González-lópez E, Gallego-delgado M, Guzzo-merello G, et al. Wild-type transthyretin amyloidosis as a cause of heart failure with preserved ejection fraction. Eur Heart J. 2015;36(38):2585-94.

51. Longhi S, Guidalotti PL, Quarta CC, et al. Identification of TTR-related subclinical amyloidosis with 99mTc-DPD scintigraphy. JACC CardiovasC Imaging. 2014;7(5):531-2

52. Castaño A, Drachman BM, Judge D, Maurer MS. Natural history and therapy of TTR-cardiac amyloidosis: emerging disease-modifying therapies from organ transplantation to stabilizer and silencer drugs. Heart Fail Rev. 2015; 20(2):163-78.

53. Ando $Y$, Ueda M. Diagnosis and therapeutic approaches to transthyretin amyloidosis. Curr Med Chem. 2012;19(15):2312-23.

54. Gertz MA, Mauermann ML, Grogan M, Coelho T. Advances in the treatment of hereditary transthyretin amyloidosis: a review. Brain Behav. 2019;9(9): e01371.

\section{Publisher's Note}

Springer Nature remains neutral with regard to jurisdictional claims in published maps and institutional affiliations.

Ready to submit your research? Choose BMC and benefit from:

- fast, convenient online submission

- thorough peer review by experienced researchers in your field

- rapid publication on acceptance

- support for research data, including large and complex data types

- gold Open Access which fosters wider collaboration and increased citations

- maximum visibility for your research: over $100 \mathrm{M}$ website views per year

At BMC, research is always in progress.

Learn more biomedcentral.com/submissions 